\title{
Mission and Aircraft Design of FLEXOP Unmanned Flying Demonstrator to Test Flutter Suppression within Visual Line of Sight
}

\author{
Stahl, Philipp ${ }^{1}$ \\ Technische Universität München, Institute of Aircraft Design, Boltzmannstr. 15, Garching bei München, 85748, \\ Germany \\ Sendner, Franz-Michael ${ }^{2}$ \\ Technische Universität München, Institute of Aircraft Design, Boltzmannstr. 15, Garching bei München, 85748, \\ Germany \\ Hermanutz, Andreas ${ }^{3}$ \\ Technische Universität München, Institute of Lightweight Structures, Boltzmannstr. 15, Garching bei München, \\ 85748, Germany \\ Rößler, Christian ${ }^{4}$ \\ Technische Universität München, Institute of Aircraft Design, Boltzmannstr. 15, Garching bei München, 85748, \\ Germany \\ Hornung, Mirko ${ }^{5}$ \\ Technische Universität München, Institute of Aircraft Design, Boltzmannstr. 15, Garching bei München, 85748, \\ Germany
}

Flexible wing structures hold the potential to enhance the performance of future aircraft. A drawback however is their reduced flutter onset speed. FLEXOP project therefore develops methods for an active suppression of flutter. To perform the risky flutter test flights required for validation, an on-purpose designed unmanned demonstrator aircraft is used. As the test flights take place in German airspace, the test flights are limited to visual line of sight range of the operator. For this uncommon approach, first a flutter test methodology has been identified and a respective mission has been designed. Looking at the mission, it appears that the aircraft design process has to focus on precise evaluation of all acceleration and deceleration procedures as well as on the achievement of low flutter onset speed, but with high fidelity in its prediction. Hence, an aircraft design toolchain was implemented that includes flutter analysis based on high detail, parameterized aero-elastic models and a mission simulation incorporating also the dynamic behavior of the main relevant aircraft systems. As this design toolchain takes only few fundamental aircraft parameters as input, it was possible to evaluate a number of different aircraft layouts for their mission performance. The resulting aircraft design yields a jet engine powered demonstrator aircraft with seven meters wingspan and $65 \mathrm{~kg}$ design mass. The flutter onset for the highly flexible wing is predicted in the $50 \mathrm{~m} / \mathrm{s}$ regime.

\section{Nomenclature}

FLEXOP = Flutter Free Flight Envelope Expansion for Economical Performance Improvement (Project Name) VLOS $=$ Visual Line of Sight

\footnotetext{
${ }^{1}$ Research Scientist, Technische Universität München, Institute of Aircraft Design, philipp.stahl@tum.de

${ }^{2}$ Research Scientist, Technische Universität München, Institute of Aircraft Design, franz.sendner@tum.de

${ }^{3}$ Research Scientist, Technische Universität München, Institute of Lightweight Structures, andreas.hermanutz@tum.de

${ }^{4}$ Research Associate, Technische Universität München, Institute of Aircraft Design, christian.roessler@tum.de

${ }^{5}$ Professor, Technische Universität München, Institute of Aircraft Design, mirko.hornung@tum.de
} 


\section{Introduction}

Flexible wing structures are a promising technology to increase the performance of future aircraft. Their structure can be realized with lower mass and their increased slenderness improves aerodynamic performance. These wings, however, are more prone to dangerous aero-elastic phenomenon like flutter. A strategy to overcome this design boundary is active flutter control. For the design of flexible wings and active flutter control systems, tools have to be developed and validated. For validation purpose, flight test data is highly desirable. Flight testing near and beyond the flutter boundary however involves high risk. The FLEXOP project therefore uses an unmanned demonstrator aircraft. The regulations for unmanned aircraft operation in Germany where the test flights shall take place limits the test mission to visual line of sight. This drastically affects the design of the flutter test mission and the aircraft design itself.

\section{Mission Design}

Flutter testing takes place during straight flight with constant altitude and speed. An excitation e.g. in form of a harsh control input is given to the aircraft and the following oscillation is measured. This procedure is repeated for several speeds below the expected flutter onset speed. The trend of the oscillation's damping for different speeds allows conclusion on the actual flutter onset speed. With the flutter suppression system activated, it is planned to exceed the natural flutter onset speed by up to $20 \%$. As these flight tests shall take place in Germany, the flutter test pattern and the aircraft itself have to be conform with German regulations for the operation of unmanned aerial systems. This implicates a take-off mass of below $150 \mathrm{~kg}$ and operation within VLOS without artificial assistance. A safe value for the visual line of sight range was determined by test flights with existing, comparable unmanned aircraft. Within this range, various flight patterns providing a longest possible straight flight path were evaluated. Fig. 1 shows an elongated circle pattern. It starts with turn at an airspeed significantly below flutter onset speed to not initiate flutter under an increased load factor. Then, the aircraft accelerates and settles the desired airspeed as fast as possible. After performing the excitation and oscillation measurements. The aircraft decelerates for the final turn to safe airspeed before the VLOS limit is reached.

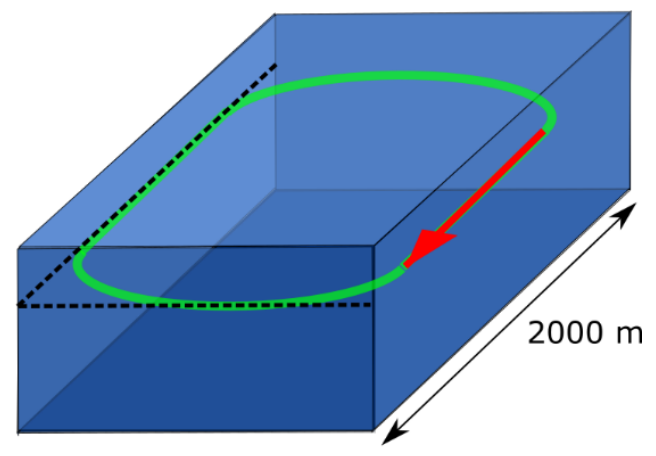

Fig. 1: Possible Test Flight Pattern (Measurement Leg Marked in Red)

\section{Aircraft Design}

To have sufficient measurement time on the straight test leg, an aircraft with high acceleration and deceleration capabilities is required. Furthermore, the flutter onset speed has to be low, nevertheless leaving enough margin for aircraft operation. An additional requirement comes from the actuators on the flutter control surfaces. Their bandwidth limits the feasible frequency of the flutter modes. Facing this constellation, an aircraft design toolchain was implemented that involves flutter evaluation based on detailed aero-elastic aircraft models already in the early design phase. Besides flutter analysis, the toolchain predicts aerodynamic performance, sizes load carrying structures, selects relevant systems from databases, estimates aircraft mass and simulates the mission performance, all by the input of few fundamental design parameters. Thus, it was possible to set up a systematic variation of these parameters to yield an aircraft layout best suitable for the mission. During the detailed design phase, the flutter behavior of the suggested layout was ascertained by more precise methods and the mission performance was monitored. Fig. 2 shows the aircraft at the end of the detailed design. 


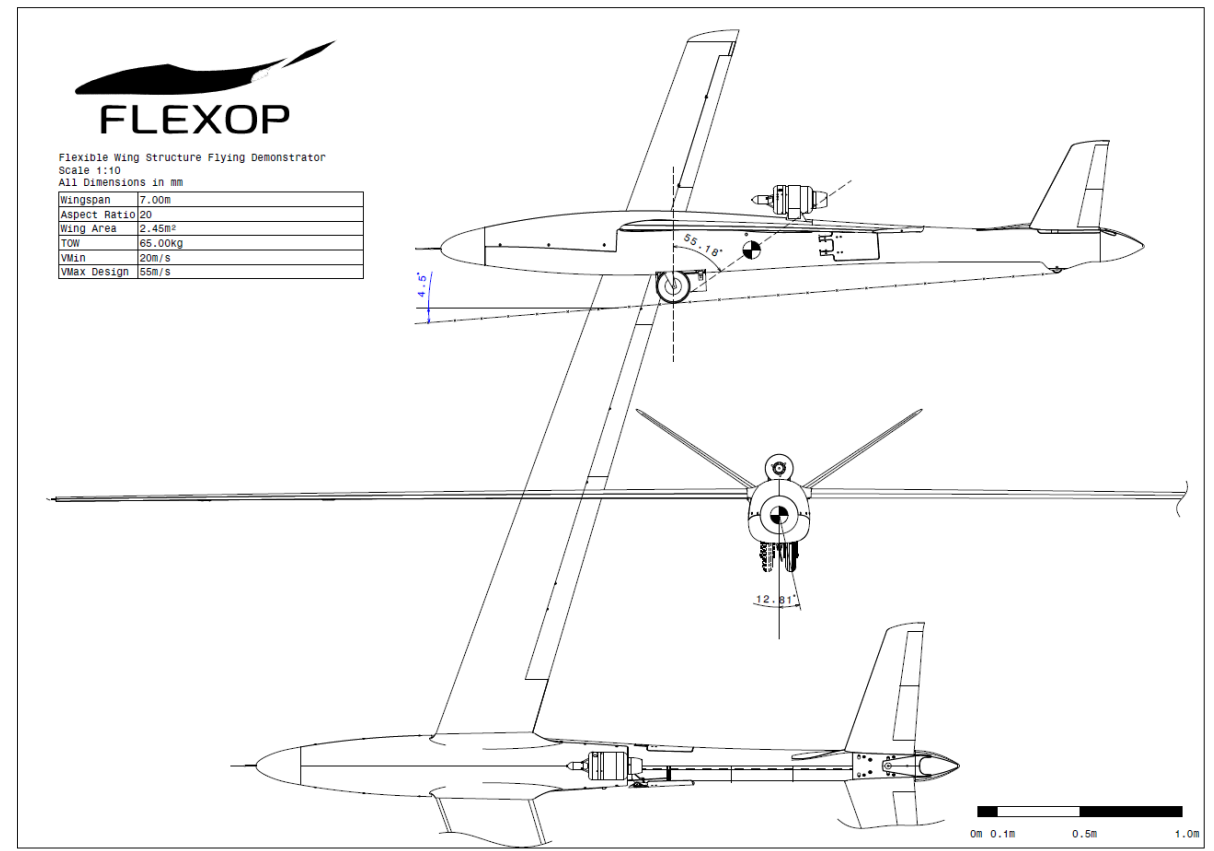

Fig. 2: Drawing of FLEXOP Demonstrator

The wing features a wing span of seven meters and an aspect ratio of 20 . With its highly flexible structure, flutter onset speeds between 45 to $50 \mathrm{~m} / \mathrm{s}$ at flutter frequencies beginning at $15 \mathrm{~Hz}$ are predicted to be achievable. For sufficient acceleration and deceleration performance, the FLEXOP demonstrator is equipped with a $300 \mathrm{~N}$ jet engine and an airbrake system. A parachute termination system as well as a redundant power supply, command and control architectures are implemented to fulfill German regulations for UAV operation.

\section{Current Status and expected Status until May 2017}

Currently, component tests with actuators and engine are conducted. With the help of rapid prototyping and 3D printing, also subsystems like recovery parachute release system and airbrakes can be tested via mock-ups in an early detail design phase. Until May 2017, the manufacturing of fuselage and empennage structure, as well as first wing structures shall be completed. The aircraft will be integrated to perform overall system tests.

\section{Acknowledgement}

The research leading to these results is part of the FLEXOP project. This project has received funding from the European Union Horizon 2020 research and innovation program under grant agreement No 636307.

\section{References}

FLEXOP Consortium. (2015). The FLEXOP Project. Retrieved from www.flexop.eu

Guffler, A. (2016). Design of Fuselage and Empennage of FLEXOP Umanned Research Aircraft in Catia V5. Munich: Institute of Aircraft Design.

Kehoe, M. W. (1995). A Historical Overview on Flight Flutter Testing. Dryden: NASA Dryden Flight Research Center.

Kolb, T. (2016). Design of a Recovery System for FLEXOP Unmanned Research Aircraft. Munich: Institue of Aircraft Design.

Sendner, F.-M. (2016). Evaluation and Selection of a Propulsion System for the FLEXOP Unmanned Research Aircraft. Munich: Institute of Aircraft Design. 